This is an Open Access article distributed under the terms of the Creative Commons Attribution-Noncommercial License, which permits unrestricted use, distribution, and reproduction in any noncommercial medium, provided the original work is properly cited.

\title{
DEVELOPMENT OF THE SAFARI INSTRUMENT SIMULATOR
}

\author{
B. Sadeghi, D.A. Naylor, and D. Hayton \\ Department of Physics \& Astronomy, University of Lethbridge, Lethbridge, Alberta, Canada
}

\begin{abstract}
Three instruments have been proposed for the JAXAESA Space Infrared Telescope for Cosmology and Astrophysics (SPICA) Mission: a mid-infrared coronagraph, a mid-infrared camera and spectrometer, and a far-infrared imaging spectrometer (SAFARI). An overview of the SAFARI instrument simulator currently being developed is presented.

The simulator will not only find use during the design phase of the instrument, but also during the evaluation of the performance of the flight model during the ground test campaigns. Through validation of the simulator by using ground test data, it will be possible to predict reliably the in-orbit performance of SAFARI. Specifically, the simulator will be used to investigate the scientific capabilities of the instrument at three resolving powers $(\lambda / \Delta \lambda=3,100$, $2000)$ over the wavelength range $30-210 \mu \mathrm{m}$, nominally separated into three sub-bands (Swinyard \& Nakagawa, 2008).
\end{abstract}

Key words: SPICA, SAFARI, Imaging Fourier Transform Spectrometer, Software Simulator

\section{Introduction - Simulator Architecture}

The SAFARI instrument simulator is based on its precursor SHIFTS, the Simulator for the Herschel Imaging Fourier Transform Spectrometer (SPIRE) developed at the University of Lethbridge (Lindner et al., 2006), which has been successfully used to predict the performance of SPIRE. The SAFARI simulator builds upon the SHIFTS concept, being modular in nature and utilizes universal time and spectral grids. The software development is carried out using the Interactive Data Language (IDL ${ }^{\circledR}$ ), which is well-suited for the array manipulation and visualization techniques required in the simulator. The architecture consists of a series of independent and self-contained modules which simulate a physical quantity within the instrument, typically output as a time-series or spectrum. By employing a well-defined set of input and output parameters for each module, this structuring ensures that a new module can be integrated easily without affecting the rest of the simulator. Subsequent changes to a module can also be implemented with ease.
Figure 1 summarizes the modular component design and the data flow scheme of the simulator. The modular components include Optics, Pointing, Mach-Zehnder imaging Fourier transform spectrometer (FTS), Poweron-Pixel, Detector and Read Out electronics. The Optics Module constitutes the skeleton of the simulator, comprised of the telescope and instrument optical components $(30+$ elements cooled to $\sim 5 \mathrm{~K})$ which are divided into three sections (pre-FTS, FTS, post-FTS) and are used to determine the radiant load on the detectors. The Pointing Module includes contributions from the telescope and the beam steering mirror. The spectrometer mechanism (FTS Module) simulates the non-uniform spatial sampling of the interferogram, which results from the variable speed of the scanning mechanism. The only terms which can give rise to a modulated component are the astronomical source and any emission from pre-FTS optics. The total power received at the detectors is computed in the Power-on-Pixel Module. The 'black-box' Detector Modules are intended to facilitate the simulation and testing of the different detector technologies (e.g. TES bolometers, kinetic induction detectors, photoconductors).

\section{Source Spectral Cube Module}

The Source Spectral Cube Module is a simulated, timeindependent, three-dimensional data cube, with two spatial coordinates and one spectral, expressing spectral intensities in units of $\mathrm{W} / \mathrm{m}^{2} / \mathrm{cm}^{-1} / \mathrm{sr}$. Point and extended sources are simulated with discrete spectral lines, continua, and combinations of both. The simulator ensures that the source spectral resolution exceeds that defined by the maximum optical path difference (OPD) within the interferometer. This will allow us to explore the ability to retrieve spectral information beyond the limit of the FTS resolution. The goal is to generate a realistic spectral profile of a synthetic molecular cloud or galaxy, to determine how well the spectrum can be reconstructed.

\section{Pointing Module}

The Pointing Module consists of two components, one is pointing of the telescope as a whole (i.e. low frequency oscillations), and the second is smaller higher frequency adjustments to the pointing provided by the beam steering mirror (BSM). Since the Herschel Space Observatory 


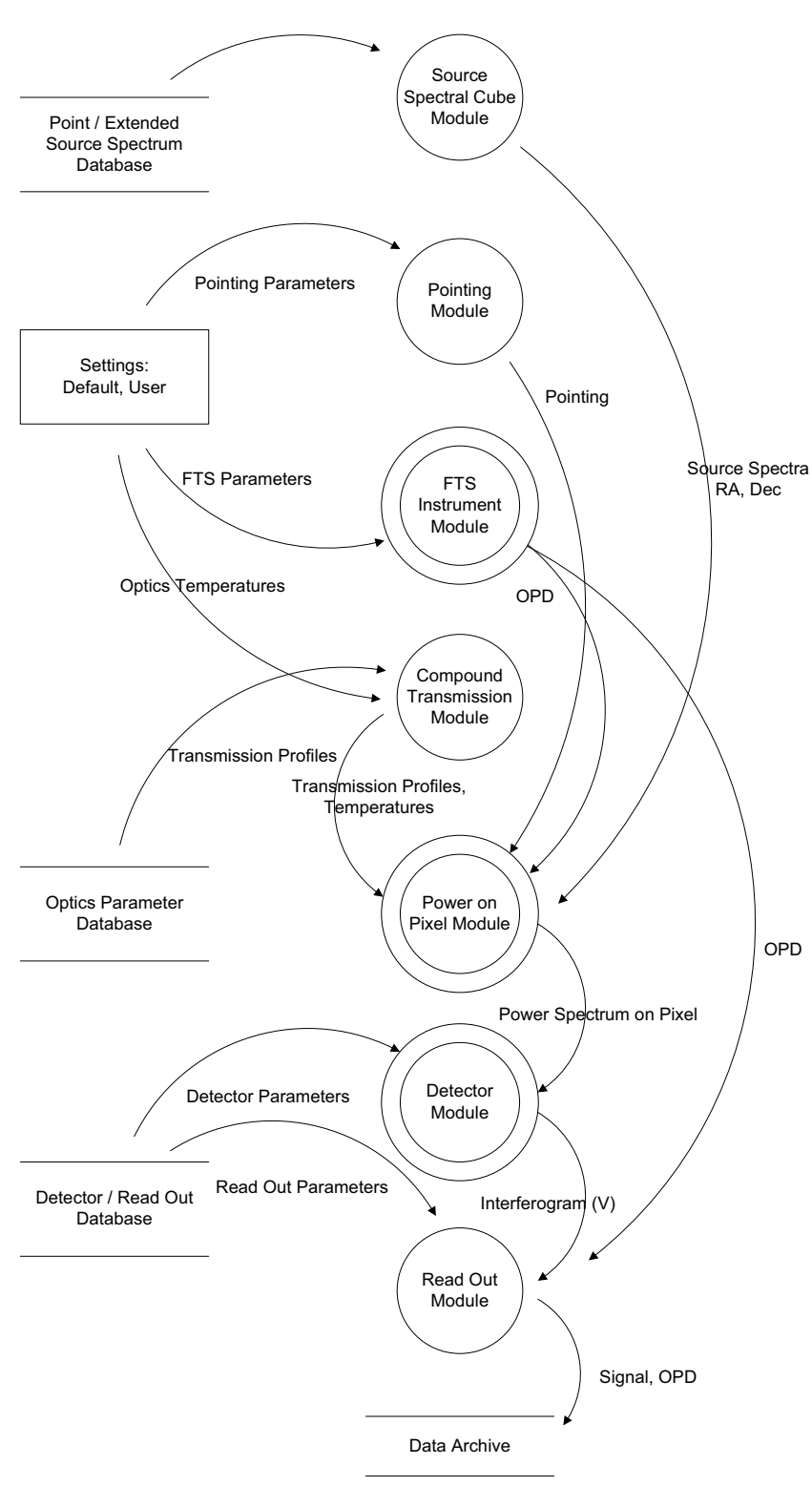

Figure 1. Modular design and data flow diagram.

(launched in May 2009) shares many features in common with SPICA, we are planning to use pointing information obtained from the Herschel mission to characterize the BSM.

\section{Optical Components}

The optical component design consists of a series of elements common to all SPICA instruments and a set belonging to SAFARI, over 30 components in all. The primary and secondary mirrors (external) will be actively cooled at a temperature of $\sim 6$ Kelvin, while the intermediate components will be cooled to $\sim 5 \mathrm{~K}$ and the detector-box ele- ments to sub-Kelvin temperatures (Nakagawa, 2008). The sequence of the components is defined by a table containing transmission/reflection spectral profiles, phase profiles, and temperature time-lines for each element. Given a sequential optics table, the Compound Transmission Module computes complex amplitude 'stack' transmission coefficient profiles for all components, divided into pre-FTS, FTS and post-FTS portions. The pre-FTS stack transmission amplitude is given by

$T_{n_{\text {pre-FTS }}}(\sigma)=\prod_{m=n+1}^{N_{\text {pre }}} t_{m}(\sigma) e^{i \phi_{m}(\sigma)}$,

where $\sigma$ is in wavenumbers, $t_{m}$ and $\phi_{m}$ are, respectively, the spectral transmission and phase profiles of the $m$-th component of the pre-FTS optics, and $N_{\text {pre }}$ is the final pre-FTS component. The FTS and post-FTS stack profiles are computed in a similar fashion. The stack profiles determine what portion of an element's blackbody self-emission contributes to the radiant load on to the detectors at a given time. The reflection/transmission and phase spectral profiles will be determined by the testing of the individual sub-system components.

\section{Spectrometer Mechanism Module}

SAFARI implements a modified Mach-Zehnder (MZ) interferometer design. In the modified MZ design, the arms of the classic MZ interferometer are folded, thus reducing the mass and volume of the device, and also allowing a single translation stage to simultaneously modulate the optical paths of the two beams. Within the spectrometer, the astronomical source beam is divided into two by the first beam splitter. The reflected and transmitted beams are directed to complementary mirrors and then onto the moving back-to-back root-top mirrors (where the optical retardation is introduced), before recombining at the second beam splitter, and subsequently being focussed onto the detectors.

In calculating the OPD between the two beams, the spectrometer simulator mimics the stage velocity of the scanning mirrors according to the spectral velocity error profiles determined during the ground test campaigns. It is common practice in Fourier spectroscopy to measure the interferogram in equal intervals of OPD. However, this is not practical for a space based mission, and as with the Herschel SPIRE FTS, SAFARI measures the detector signal and the stage position at different times and interpolates them on to a common time grid, from which the detector signal can be constructed as a function of the OPD. The resulting time-series are fed to the Power-onPixel Module where the phase modulations are performed.

\section{Power on Pixel Module}

In order to compute the astronomical power received by the detectors from the field-of-view (FOV), Power-on-Pixel 
first determines the FOV according to the telescope's pointing coordinates. A wavelength dependent FOV is introduced to account for the effects of diffraction. In this way, Power-on-Pixel sums over the spatial coordinates and returns the total power delivered for the FOV corresponding for a particular wavelength.

The self-emission of all optical components positioned after the pre-FTS portion of the design are unmodulated, while the astronomical source and pre-FTS optics emissions experience modulation due to the varying OPD. The E-field on to the FTS can be expressed as

$E_{\text {pre }}=\left[\frac{2}{c \epsilon_{0}}\left(P_{s k y}\left|T_{1_{\text {pre-FTS }}}\right|^{2}+\sum_{m=1}^{N_{\text {pre }}} B_{m}\left|T_{m_{\text {pre }}}\right|^{2}\right)\right]^{\frac{1}{2}}$

where the frequency dependence has been omitted for clarity. $P_{s k y}(t, \sigma)$ is the power received from the astronomical source, with $T_{1_{\text {pre-FTS }}}$ its corresponding transmission to the FTS, $B_{m}(t, \sigma)$ is the blackbody emission of the $m$-th optical component, and $T_{m}(\sigma)$ is the corresponding transmission amplitude. The wavelength dependent throughput is also taken into account, but is omitted for clarity. The phase modulations are carried out at the second beam splitter (BS) of the FTS, with the dual output E-fields computed as

$$
\begin{aligned}
& E_{\uparrow}=E_{\text {pre }}\left(R_{1_{i}} e^{i \phi_{r_{i}}} R_{1_{c}} e^{i \phi_{r_{c}}} e^{i \sigma z}+T_{1} e^{i \phi_{t}} T_{2} e^{i \phi_{t}} e^{-i \sigma z}\right) \\
& E_{\downarrow}=E_{\text {pre }}\left(R_{1_{i}} e^{i \phi_{r_{i}}} T_{2} e^{i \phi_{t}} e^{i \sigma z}+T_{1} e^{i \phi_{t}} R_{2_{i}} e^{i \phi_{r_{i}}} e^{-i \sigma z}\right),
\end{aligned}
$$

where $T_{1,2}(\sigma)$ are the transmission amplitudes of the first and second $\mathrm{BS}, R_{1,2_{i, c}}(\sigma)$ are the BS reflection amplitudes of the inductive and capacitive sides, with $\phi_{t, r}$ being their corresponding phases, $\sigma$ is the spectral grid in wavenumbers, and $z(t)$ is the OPD. The unmodulated portion of the total power, $P_{\text {post }}(t, \sigma)$, is combined with the modulated to determine the total power of the two output ports incident on a detector pixel at a given time:

$$
\begin{aligned}
& P_{\text {post }}=\sum_{m=m_{F T S}}^{N_{\text {post-FTS }}} B_{m}\left|T_{m}\right|^{2} \\
& P_{\uparrow}=\frac{c \epsilon_{0}}{2}\left|E_{\uparrow}\right|^{2}+P_{\text {post }} \\
& P_{\downarrow}=\frac{c \epsilon_{0}}{2}\left|E_{\downarrow}\right|^{2}+P_{\text {post }} .
\end{aligned}
$$

\section{Detector Module}

The power spectrum integration is carried out in the Detector Module. First the detector coupling and quantum efficiency are applied to the incoming power spectrum. The product of the detector spectral responsivity $[\mathrm{V} / \mathrm{W}]$ and the incident power is then integrated across the spectral band, resulting in the interferogram

$V(t)=\int P(t, \sigma) R(\sigma) d \sigma$.

Initial noise-equivalent-power calculations are performed assuming that the detector will be photon noise limited (other noise sources such as Johnson, phonon, etc., are detector-specific and will be added at this stage). Regardless of the choice of the detector technology, the combination of thermal, temporal and electrical responses must be taken into account. These are combined into a detectorspecific impulse response function, which is convolved with the time-varying power incident on the detector to simulate the response of the detection system as the spectrometer is scanned. Finally, the detector voltages are output to the Read Out Module for re-sampling.

Since the different detector technology candidates have unique couplings, spectral responsivities, quantum efficiencies, and frequency response characteristics, a unique detector module is required for each candidate detector. The interchangeable nature of the Detector Modules facilitates the performance investigation and testing of the detector candidates.

\section{TES BOLOMETERS}

Transition Edge Sensors (TES) bolometers form the baseline detector for the SAFARI instrument. TES devices are a type of bolometer consisting of superconducting thin films that are biased at the midpoint of the normal to superconducting transition, where they are intrinsically linear devices. With a very high temperature coefficient of resistance, they experience large changes in resistance with small changes in temperature, resulting in an extremely sensitive device. A change in incident photon flux results in a change in the current through the TES to maintain the operating point of the detector at the midpoint of the transition. This current represents the measured signal (Irwin \& Hilton, 2005).

Our group has used FTS for several years and is developing an imaging FTS for use with the SCUBA-2 TES bolometer array (Naylor \& Gom, 2004) currently being commissioned at the James Clerk Maxwell Telescope.

Figure 2 displays the band coverage and predicted point source sensitivities for spectroscopic observations possible with SAFARI, determined using the simulator. The state of the art performance (SOAP) is based on $\mathrm{Si}: \mathrm{Sb} \mathrm{BIB}$ and Ge:Ga photoconductors, and the Goal performance is based on TES bolometers, with the zodiacal background taken as the limiting photon noise (Griffin, 2009). The zodiacal light places a fundamental limit on what can be achieved from any space-born far-infrared telescope. Also given are the estimated sensitivities of the Herschel SPIRE and PACS instruments (Griffin et al., 2006), showing that there exists two orders of magnitude improvement in sensitivity as a direct result of having an actively cooled telescope. The challenge is to develop detectors that can exploit this low-background environment.

\section{Read Out Module}

The last step in the simulator is the production of a synthetic data product defined by the detector voltages and 


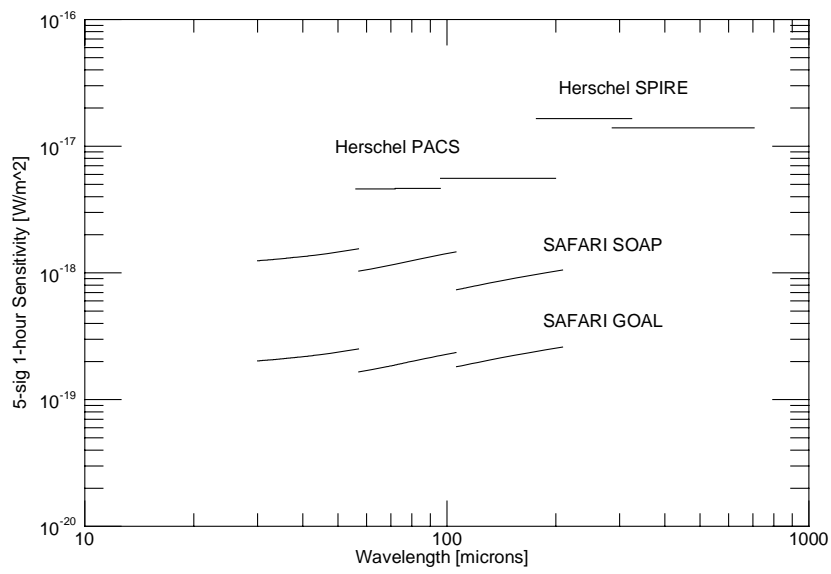

Figure 2. Predicted spectroscopic performance of SAFARI.

OPD as a function of time. It should be noted that the detector voltages and OPD will be sampled on different time grids due to limitations of on-board electronics and telemetry. The resulting time-series are saved to an external electronic file in FITS format.

\section{VERIFICATION}

Our group is developing a test facility in the AIG labs to evaluate the performance of proposed detector technologies and cryogenic components of the FTS proposed for the SAFARI instrument. While the TES technology is maturing, we have been studying Ge:Ga photoconductors (PC) supplied by the Herschel PACS instrument team at the Max Planck Institute of Extraterrestrial Physics, Munich. The Ge:Ga PCs require operation at $1.6 \mathrm{~K}$ and are responsive from $45-110 \mu \mathrm{m}$ and $110-210 \mu \mathrm{m}$, for the unstressed and stressed cases, respectively (Rosenthal et al., 2000). While these detectors are unlikely to be selected for the SPICA mission, they provide us with unique data with which to validate the simulator.

\section{Conclusion}

We are developing a simulator for the proposed SAFARI instrument. The simulator extends earlier work by Lindner et al., which was used successfully to model the SPIRE instrument. Since SAFARI and SPIRE share many aspects in common, we hope to use real data to model, realistically, the performance of SPICA. We anticipate that the simulator will find a range of uses throughout the lifetime of the project, from studying trade-offs in the design, interpreting ground test data, to predicting the in-orbit performance of the instrument.
ACKNOWLEDGEMENTS

This work is funded with support by the Canadian Space Agency (CSA), in support of the missions selected for study by the European Space Agency (ESA) Cosmic Vision Program, and NSERC.

\section{REFERENCES}

Griffin, M. 2009, "SAFARI Sensitivity Model," SPICA Int. Consortium Doc.

Griffin, M., Abergel, A., Ade, P., André, P., Baluteau, J.P., Bock, J., Franceschini, A., Gear, W., Glenn, J., Griffin, D., King, K., Lellouch, E., Naylor, D.A., Olofsson, G., Perez-Fournon, I., Rowan-Robinson, M., Saraceno, P., Sawyer, E., Smith, A., Swinyard, B., Vigroux, L., Wright, G. 2006, Proc. SPIE 6265, 62650A

Irwin, K.D., Hilton, G.C. 2005, Topics Appl. Phys. 99, 63 Lindner, J.V., Naylor, D.A., Swinyard, B.M. 2006, Proc. SPIE 6265

Nakagawa, T. 2008, 19th Int. Symp. Space THz Tech.

Naylor, D.A., Gom, B.G. 2004, Proc. SPIE 5159, 91

Rosenthal, D., Beeman, J.W., Geis, N., Looney, L.W., Poglitsch, A., Park, W.K., Raab, W., Urban, A. 2000, Proc. SPIE 4014, 156

Swinyard, B.M., Nakagawa, T. 2008, Exp. Astron. 23, 193 